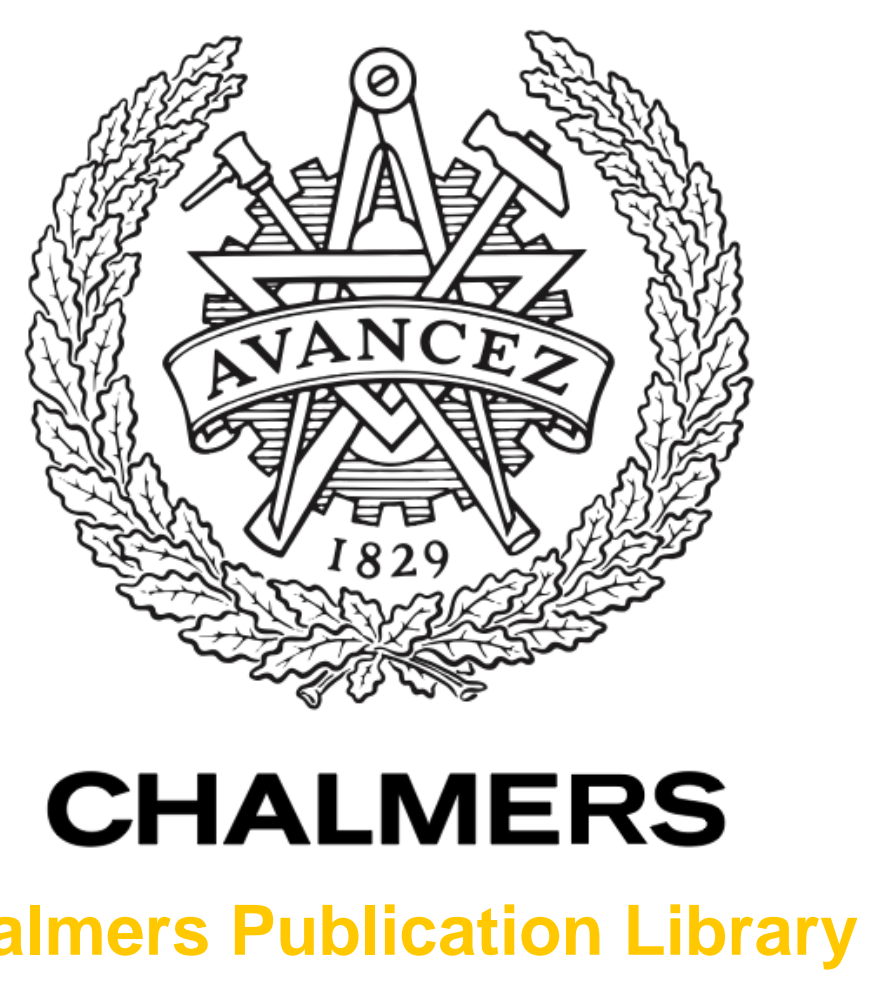

Chalmers Publication Library

\title{
A 3D/2D comparison between heterogeneous mesoscale models of concrete
}

This document has been downloaded from Chalmers Publication Library (CPL). It is the author's version of a work that was accepted for publication in:

Multi- Scale Modeling and Characterization of Infrastructure Materials. RILEM Bookseries (ISSN: 2211-0844)

Citation for the published paper:

Nilenius, F. ; Larsson, F. ; Lundgren, K. et al. (2013) "A 3D/2D comparison between heterogeneous mesoscale models of concrete". Multi- Scale Modeling and Characterization of Infrastructure Materials. RILEM Bookseries, vol. 8 pp. 249-259.

http://dx.doi.org/10.1007/978-94-007-6878-9_18

Downloaded from: http://publications.lib.chalmers.se/publication/178917

Notice: Changes introduced as a result of publishing processes such as copy-editing and formatting may not be reflected in this document. For a definitive version of this work, please refer to the published source. Please note that access to the published version might require a subscription.

Chalmers Publication Library (CPL) offers the possibility of retrieving research publications produced at Chalmers University of Technology. It covers all types of publications: articles, dissertations, licentiate theses, masters theses, conference papers, reports etc. Since 2006 it is the official tool for Chalmers official publication statistics. To ensure that Chalmers research results are disseminated as widely as possible, an Open Access Policy has been adopted.

The CPL service is administrated and maintained by Chalmers Library. 


\title{
A 3D/2D Comparison between Heterogeneous Mesoscale Models of Concrete
}

\author{
Filip Nilenius ${ }^{1,2, \star}$, Fredrik Larsson ${ }^{2}$, Karin Lundgren $^{1}$, \\ and Kenneth Runesson ${ }^{2}$ \\ 1 Department of Civil and Environmental Engineering, Chalmers University of \\ Technology, Göteborg, Sweden \\ 2 Department of Applied Mechanics, Chalmers University of Technology, \\ Göteborg, Sweden
}

\begin{abstract}
A model for 3D Statistical Volume Elements (SVEs) of mesoscale concrete is presented and employed in the context of computational homogenization. The model is based on voxelization where the SVE is subdivided into a number of voxels (cubes) which are treated as solid finite elements. The homogenized response is compared between 3D and 2D SVEs to study how the third spatial dimension influence the over-all results. The computational results show that the effective diffusivity of the $3 \mathrm{D}$ model is about 1.4 times that of the $2 \mathrm{D}$ model.
\end{abstract}

\section{Introduction}

Computational homogenization is a well known technique to account for material heterogeneities while keeping computational costs at a moderate level. One benefit of this technique is that it enables to determine bulk properties of a material a priori in the case of linear subscale properties. This is beneficial if the macroscopic behaviour of a material is sought and the subscale features is highly complex. In case of non-linear material behaviour the subscale response needs to be determined concurrently during the computations in a nested fashion using a $\mathrm{FE}^{2}$-approach. Furthermore, the technique opens up to "virtual testing" of new materials.

Concrete has a highly heterogeneous and random material structure at multiple length scales. Thus, considerable computational work on the effects of its heterogeneity has been done for this material, see eg. Kim and Al-rub [10], Idiart et al. 9] and Wang and Ueda [13].

However, most computational work reported in the literature deals with $2 \mathrm{D}$ models of concrete and any $3 \mathrm{D}$ feature that might be of importance is naturally not accounted for in these instances. A natural evolution in the development of computational homogenization is thus to account for all three spatial dimensions to obtain numerical results that potentially better reflect the real material behaviour.

\footnotetext{
* Corresponding author.
} 
This paper presents a 3D heterogeneous mesoscale model of concrete and compares computational homogenization between 2D and 3D models to study how the third spatial dimension contributes to the homogenized results. This is done in the context of mass diffusion; thus, the effective diffusivity of concrete is the studied material property in this work.

\section{Mesoscale SVE}

The concept of Statistical Volume Element (SVE) is fundamental to computational homogenization techniques as it is the foundation on which all computational work is built. The SVE is expected to contain all material heterogeneities in a statistical manner and tends to become an Representative Volume Element (RVE) only when it is sufficiently large, i.e. when the influence of boundary conditions are sufficiently small, see Ostoja-Starzewski [12]. The RVE, in turn, is used to extract the sought macroscopic material properties.

In this work, the mesoscale material constituents are the cement paste matrix, aggregates and Interfacial Transition Zone (ITZ). The aggregates are modelled as spheres with random spatial distribution enfolded by an interface layer of ITZ. The cement paste matrix is considered homogeneous, i.e. cracks are not accounted for.

\subsection{Sieve Curve}

The aggregates in the SVE can be of arbitrary size and quantity. The volume fraction of aggregates is defined as

$$
n_{\mathrm{a}}=\frac{V_{\mathrm{a}}}{V_{\mathrm{SVE}}} \quad n_{\mathrm{a}} \in[0,1],
$$

where $V_{\bullet}$ is the volume and sub-index 'a' denotes aggregate. The SVE model implementation allows for any realistic $n_{\mathrm{a}}$ to be used as input. Any sieve curve is possible to have as input for the SVE realization and we have the requirement that

$$
\sum_{i=0}^{N} n_{\mathrm{a}, i}=n_{\mathrm{a}},
$$

where $n_{\mathrm{a}, i}$ denotes the volume fraction of aggregate size $i$ and $N$ denotes the total number of particles sizes included in the specified sieve curve. Both $N$ and $n_{\mathrm{a}, i}$ can be chosen freely given that the constraint in Eq. (2) is fulfilled.

\subsection{Algorithm for Generating SVEs}

The algorithm for generating SVEs is given below as pseudo-code and has been implemented in MATLAB. The algorithms assures that no aggregates 


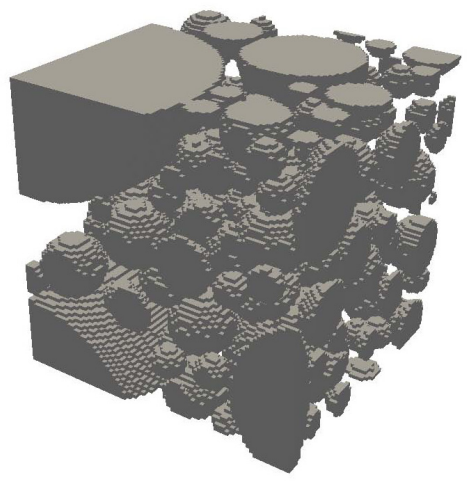

(a) $L_{\square}=2 \mathrm{~cm}$

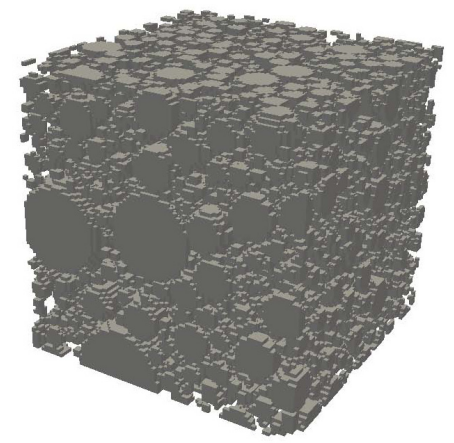

(c) $L_{\square}=6 \mathrm{~cm}$

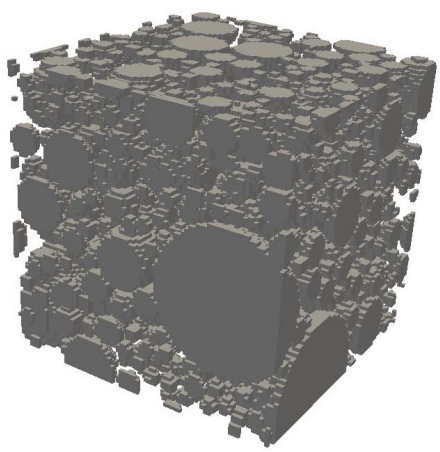

(b) $L_{\square}=4 \mathrm{~cm}$

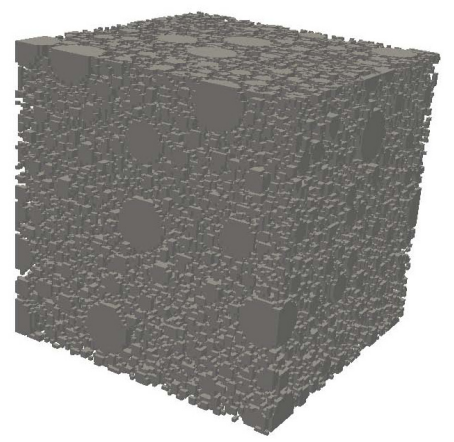

(d) $L_{\square}=10 \mathrm{~cm}$

Fig. 1 Example SVEs all having the same sieve curve with $n_{\mathrm{a}} \approx 0.45 . L_{\square}$ refers to the side lenght of the SVE.

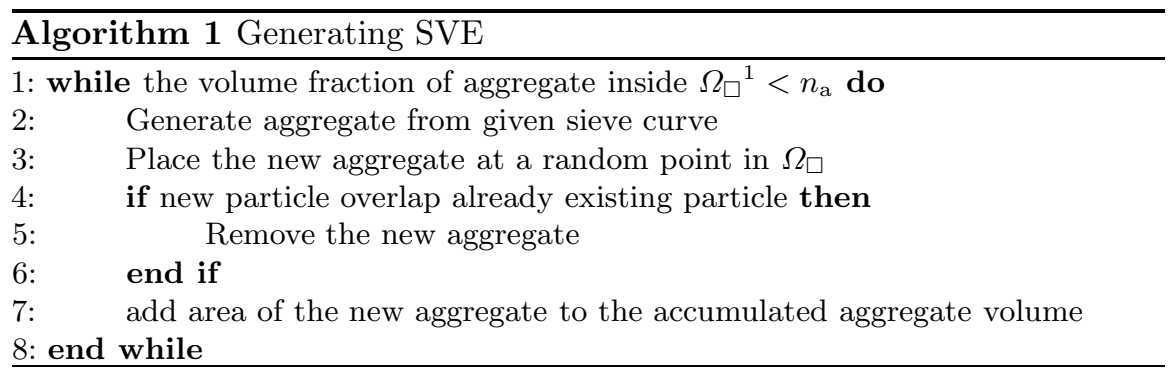

overlap once the algorithm has completed. In Figure 1, four example SVEs produced by the algorithm are show.

\footnotetext{
$\overline{1}$ The SVE domain is denoted $\Omega_{\square}$.
} 


\section{FE Discretization of SVE}

The spatial discretization technique is based on voxelization to create a structured grid of equally sized voxels. The concept is to subdivide a cubic body into a number of smaller cubes (voxels) which are considered solid finite elements. This approach was used by Bentz et al. [2] and Garboczi [5] in the context of analytical homogenization of heterogeneous concrete and later adapted by Hain and Wriggers [6, 7] for elasticity problems and computational homogenization. Other discretization approaches for 3D domains have been devised in the literature: both Caballero et al. [4] and Asahina and Bolander [1] used Voronoi tessellation where the aggregates were based on a polyhedron representation to generate 3D SVEs.

Material properties of the finite elements are determined by looping over all elements and for each element measure its distance to the center of all aggregates (spheres). If the distance is greater that the radius of any of the aggregates then the elements is located in the cement paste matrix and can be assigned its proper material property. If the distance is smaller than the radius then the element sits inside an aggregate.

\section{ITZ Implementation}

Experimental results by Hedenblad [8] indicate that the effective, or volume average, diffusivity of concrete slightly increase with increasing aggregate content, alternatively remains unchanged. This fact implies that the ITZ has a non-negligible effect on the diffusivity of concrete.

As the SVEs are discretisized into a structured grid, a certain number of voxels end up right at the interface between the cement paste matrix and aggregate, see Figure 2.

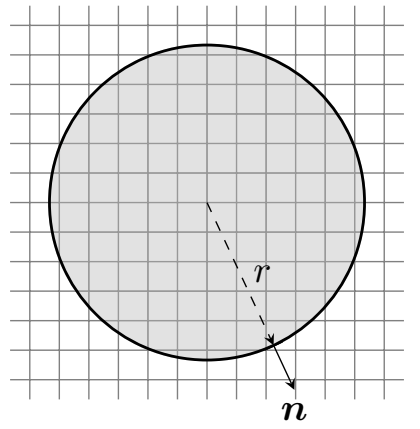

(a) 2D analogy: circle discretisized into a structured grid.

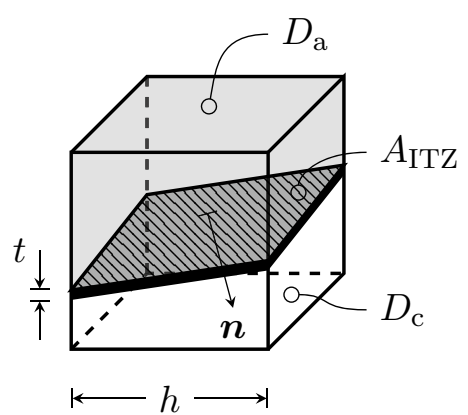

(b) Interface voxel.

Fig. 2 Interface voxel (right) containing all three mesoscale constituents located at the surface of an aggregate (left) 
Consequently, these interface voxels will contain all three mesoscale materials; cement paste, aggregate and ITZ. For these voxels, we employ an anisotropic Voigt assumption on the over-all diffusivity, expressed as

$$
\overline{\boldsymbol{D}}=\frac{V_{\mathrm{a}} D_{\mathrm{a}}+V_{\mathrm{c}} D_{\mathrm{c}}}{V_{\mathrm{a}}+V_{\mathrm{c}}} \boldsymbol{I}+\frac{A_{\mathrm{ITZ}} D_{\mathrm{ITZ}} t}{V_{\mathrm{a}}+V_{\mathrm{c}}}(\boldsymbol{I}-\boldsymbol{n} \otimes \boldsymbol{n}),
$$

where $D_{\bullet}$, are material diffusivities 1 . Generally, we have that $D_{\text {a }} \ll D_{\text {c }} \ll$ $D_{\text {ITZ }}$ and for this work we assume that $D_{\mathrm{a}}=0 \mathrm{~cm}^{2} / \mathrm{s}$ and we set $D_{\mathrm{c}}=$ $1 \mathrm{~cm}^{2} / \mathrm{s}$. The unknown parameters in Eq. (3) are the thickness, $t$, and diffusivity, $D_{\text {ITZ }}$, of the ITZ. Computationally, it is the product of these two, $D_{\text {ITZ }} \times t$, that become the model parameter.

There are several ways in which the aggregate interface surface, $A_{\text {ITZ }}$, can cut through the voxel and the intersection points between the surface and the line segments of the voxel can range from 3 to 6 . The gray and white aggregate and cement paste volumes in Figure $2 \mathrm{~b}$ become convex hulls, see Berg et al. [3], spanned by these intersection points and corner nodes, and are computed by utilizing this geometrical property. The interface area, $A_{\text {ITZ }}$, is determined in a similar fashion.

\section{First Order Homogenization}

We wish to determine the difference between 3D and 2D SVEs in terms of homogenized response. This is done by starting from the fully resolved, stationary, boundary value problem

$$
\boldsymbol{\nabla} \cdot \boldsymbol{J}=0 \quad \forall \boldsymbol{x} \in \Omega
$$

for which all material heterogeneities are embedded in $\Omega \subset \mathbb{R}^{3}$. Here, $\boldsymbol{\nabla}$ is the nabla operator and $\boldsymbol{J}(\boldsymbol{x})$ is the flux of some generic physical quantity. The corresponding weak form reads

$$
\int_{\Omega} \nabla \delta \phi \cdot \boldsymbol{J}=\int_{\Gamma_{\mathrm{N}}} \delta \phi \boldsymbol{J} \mathrm{d} \Gamma,
$$

for suitable test function $\delta \phi$ that is sufficiently regular.

Homogenization implies that the integrands of the volume integrals in Eq. (5) are replaced by space-averages on RVEs, i.e.

$$
\int_{\Omega} \boldsymbol{\nabla} \delta \phi \cdot \boldsymbol{J} \mathrm{d} \Omega \longrightarrow \int_{\Omega}\langle\boldsymbol{\nabla} \delta \phi \cdot \boldsymbol{J}\rangle_{\square} \mathrm{d} \Omega,
$$

where $\langle\bullet\rangle_{\square}$ denotes the volume average

$$
\langle\bullet\rangle_{\square} \stackrel{\text { def }}{=} \frac{1}{\left|\Omega_{\square}\right|} \int_{\Omega_{\square}} \bullet \mathrm{d} \Omega_{\square},
$$

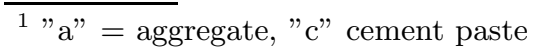


on the RVE that occupies the domain $\Omega_{\square}$ centered at the macroscale position $\overline{\boldsymbol{x}} \in \Omega$; hence, $\langle\boldsymbol{x}-\overline{\boldsymbol{x}}\rangle_{\square}=0$.

We assume first order homogenization defined by the split of a scalar field $\phi$ within $\Omega_{\square}$ into the macroscale and fluctuation part:2 as follows:

$$
\phi(\boldsymbol{x} ; \overline{\boldsymbol{x}})=\phi^{\mathrm{M}}(\boldsymbol{x} ; \overline{\boldsymbol{x}})+\phi^{\mathrm{s}}(\boldsymbol{x}) .
$$

The macroscale part varies linearly as

$$
\phi^{\mathrm{M}}(\boldsymbol{x} ; \overline{\boldsymbol{x}})=\bar{\phi}(\overline{\boldsymbol{x}})+\overline{\boldsymbol{g}}_{\phi}(\overline{\boldsymbol{x}}) \cdot[\boldsymbol{x}-\overline{\boldsymbol{x}}] \quad \forall \boldsymbol{x} \in \Omega_{\square},
$$

with $\overline{\boldsymbol{g}} \stackrel{\text { def }}{=} \overline{\boldsymbol{\nabla}} \bar{\phi}$. Hence, we obtain $\nabla \phi^{\mathrm{M}}(\overline{\boldsymbol{x}} ; \overline{\boldsymbol{x}})=\overline{\boldsymbol{g}}(\overline{\boldsymbol{x}})$ constant within $\Omega_{\square}$. We then obtain for any macroscale point $\overline{\boldsymbol{x}} \in \Omega$ the identity

$$
\langle\nabla \delta \phi \cdot \boldsymbol{J}\rangle_{\square}=\delta \overline{\boldsymbol{g}} \cdot \overline{\boldsymbol{J}} \quad \text { with } \quad \overline{\boldsymbol{J}}_{\phi}=\langle\boldsymbol{J}\rangle_{\square} .
$$

In order to compute the homogenized flux quantity $\overline{\boldsymbol{J}}$, it is necessary to compute the fluctuation field $\phi^{\mathrm{s}}$ as well as the spatial gradient on the subscale: $\boldsymbol{g}^{\mathrm{s}} \stackrel{\text { def }}{=} \boldsymbol{\nabla} \phi^{\mathrm{s}}$. This is done on each RVE with given prolongation conditions. Here, we choose Dirichlet boundary conditions, i. e. $\phi^{\mathrm{s}}=0$ on $\Gamma_{\square}$, whereby the pertinent RVE-problem becomes: For given values of the macroscale variables $\bar{\phi}$ and $\overline{\boldsymbol{g}}$, compute the subscale field $\phi^{\mathrm{s}}$ that satisfy the system

$$
\left\langle\nabla\left(\delta \phi^{\mathrm{s}}\right) \cdot \boldsymbol{J}\right\rangle_{\square}=0
$$

for all possible $\delta \phi^{\mathrm{s}}$ that are sufficiently regular and which vanish on $\Gamma_{\square}$. We note that the macroscale prolongations $\phi^{\mathrm{M}}$ are completely defined (varies linearly) on $\Omega_{\square}$.

Our objective is to determine the mapping

$$
L_{\square} \mapsto\langle\boldsymbol{J}\rangle_{\square}\left(L_{\square}\right)
$$

and to study how this mapping differs between 3D and 2D SVEs of mesoscale concrete models. This is done by solving Eq. (12) given a macroscale gradient $\overline{\boldsymbol{g}}=\left[\begin{array}{lll}-1 & 0 & 0\end{array}\right]^{\mathrm{T}}$ for SVEs of varying $L_{\square}$.

\section{$6 \quad 2 \mathrm{D}$ SVEs}

The 2D SVEs are generated from cutting a 3D SVE into a number of 2D slices, see Figure 3. The same boundary conditions are applied to the 3D and $2 \mathrm{D}$ SVEs. The mean values of all slices are then compared to the single result obtained for the corresponding 3D SVE. In this way, the $2 \mathrm{D} / 3 \mathrm{D}$ comparison becomes meaningful since comparison is made on the same topology.

\footnotetext{
${ }^{2}$ Superscripts $M$ denotes macroscale, whereas $s$ denotes subscale.
} 


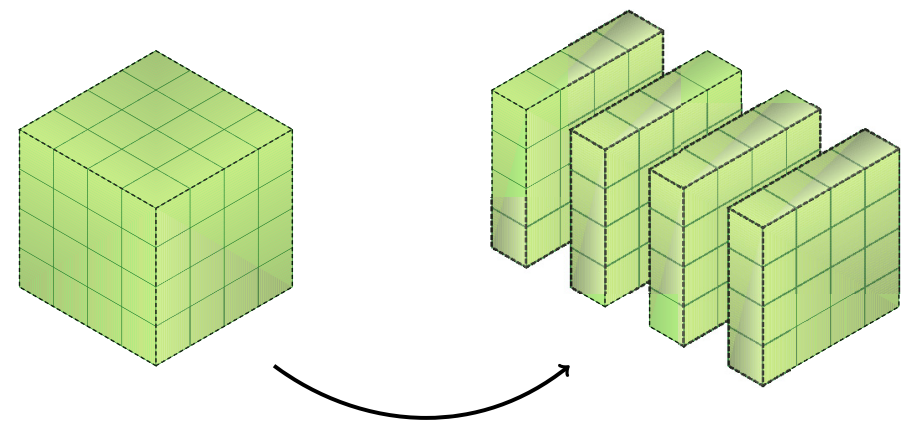

Fig. 3 3D SVE cut into 2D slices

\section{Numerical Examples}

\section{$7.13 D / 2 D$ Comparison}

The numerical example compares the first component of the homogenized flux tensor, $\langle\boldsymbol{J}\rangle_{1}$, as a function of the SVE size 3 denoted $L_{\square}$. The RVE size based on the numerical results becomes $L_{\mathrm{RVE}} \stackrel{\text { def }}{=} 10 \mathrm{~cm}$ since convergence has been reached for this value of $L_{\square}$. The cement paste was assigned a unit diffusivity of $D_{\mathrm{c}}=1 \mathrm{~cm}^{2} / \mathrm{s}$, for simplicity, and $D_{\mathrm{a}}=0 \mathrm{~cm}^{2} / \mathrm{s}$. The ITZ layer is omitted in the example.

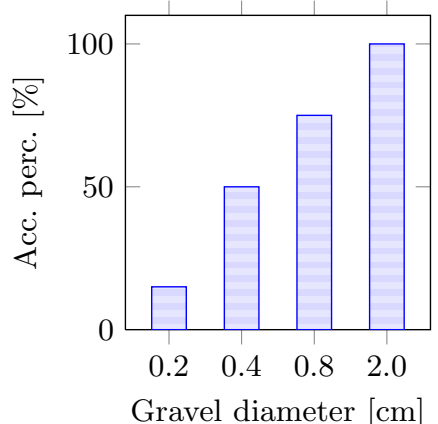

Fig. 4 Sieve curve SVEs used in the numerical example

Forty 3D SVEs were generated for each $L_{\square}$ all having an aggregate volume fraction, $n_{\mathrm{a}}$, of 0.45 with the sieve curve shown in Figure 4. Each 3D SVE was cut into $692 \mathrm{D}$ slices. Thus, $\mu_{2 \mathrm{D}}$ is the mean value of $40 \times 69=2760 \mathrm{SVE}$ slices for each $L_{\square}$.

3 "size" refers to side length of the cube. 


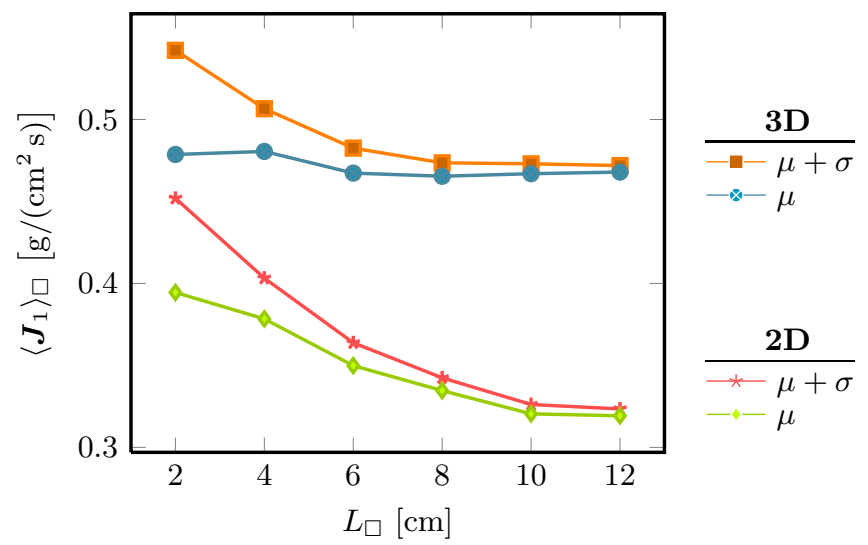

Fig. 5 Homogenized flux versus size of SVE. Average values denoted $\mu$ and corresponding standard deviations denoted $\sigma$.

The results are shown in Figure 5; they show that the 2D results in general are lower than the corresponding ones for $3 \mathrm{D}$. The reason for this is probably that the out of plane solution in the $2 \mathrm{D}$ case is locked; hence, for the $2 \mathrm{D}$ case the diffusion substance has only two directions (over or under) to bypass an aggregate but in the 3D case a third direction (around) is enabled. For $L_{\square}=10 \mathrm{~cm}, \mu_{2 \mathrm{D}} \approx 0.7 \mu_{3 \mathrm{D}}$; i.e. the $2 \mathrm{D} \mathrm{SVE}$ homogenized diffusivity is about $70 \%$ of the corresponding $3 \mathrm{D}$ value.

\subsection{The Effect of ITZ}

The same computations as $\square-\square$ and $[\bar{E}$ in Figure 5 were carried out again now including the ITZ in the mesoscale model and the results are shown in Figure 6.

The results in Figure 6 show that it is possible to increase the effective diffusivity by utilizing the ITZ implementation as a model parameter. The results also show the robustness of the implementation as the mean values, $\square$ in practice become independent of the SVE size, $L_{\square}$. Only the spread in results, $\square$ varies and decreases with increasing $L_{\square}$, as expected.

The value $D_{\text {ITZ }} \times t=0.15 D_{\text {c }}$ has manually been calibrated such that $\left\langle\boldsymbol{J}_{1}\right\rangle_{\square}$ gets close to a value of $1 \mathrm{~g} /\left(\mathrm{cm}^{2} \mathrm{~s}\right)$, i.e. corresponding to a diffusivity of pure cement paste. The outcome of this numerical example is that the over-all effect of the aggregates is that they do not change the diffusivity of the bulk material. This is achieved by the implementation of ITZ in the model. In this way, the product $D_{\text {ITZ }} \times t$ becomes a useful tool for calibrating the model with experimental data. 


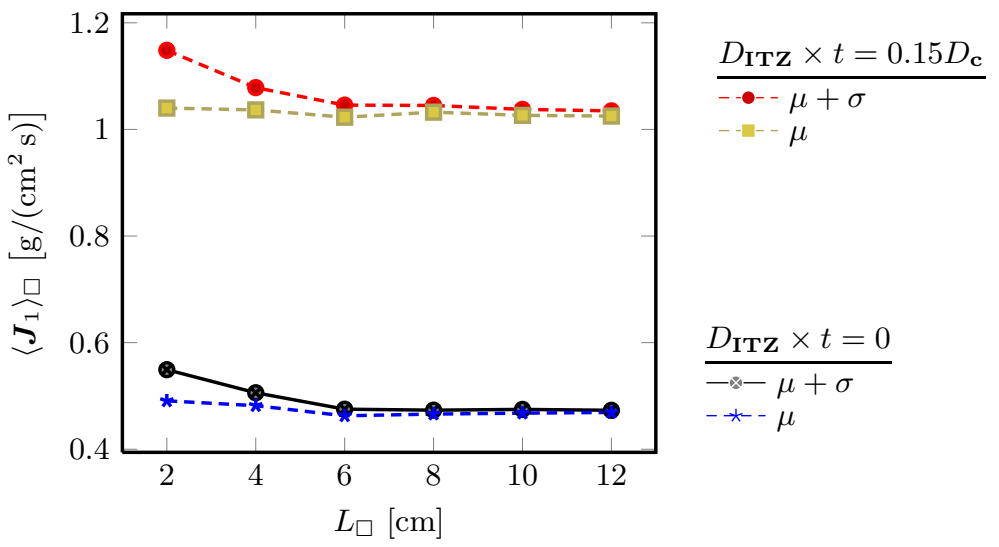

Fig. 6 Numerical results with ITZ included in the SVEs. The results for $D_{\text {ITZ }} \times t=0$ are the same as those presented in Figure $5 .\left\langle\boldsymbol{J}_{1}\right\rangle=1 \mathrm{~g} /\left(\mathrm{cm}^{2} \mathrm{~s}\right)$ corresponds to an effective diffusivity equal to that of pure cement.

\subsection{Computational Time}

The computational work for this paper has been carried out on a standard laptop machine having $4 \mathrm{~GB}$ of RAM with a dual-core processor running Windows 7. The implementation is made in MATLAB and is parallelized to reduce computational time.

The average computational time for one 3D SVE is approximately $20 \mathrm{~min}$, and approximately $0.5 \mathrm{~min}$ for one 2D SVE, but since the multiple SVEs can be run in parallel the total time is governed by the number of processors available.

\section{Conclusions and Outlook}

A 3D model of heterogeneous mesoscale concrete was presented and employed in the context of computational homogenization. The algorithm on which the model is based generates a random structure of aggregates embedded in cement paste. Any sieve curve is possible as input for the algorithm and any (realistic) aggregate content is possible to obtain.

The numerical examples presented in the paper shows that the difference between a $3 \mathrm{D}$ and $2 \mathrm{D}$ model in terms of effective diffusivity differ approximately by a factor 0.7 . The numerical results further show that the ITZ implementation is robust and can be used to calibrate/adjust the effective diffusivity of cement with embedded aggregates in it, i.e. concrete. With the ITZ included in the model, it is possible to increases the effective diffusivity of concrete in an easy and consistent manner.

The next step is to use 3D SVEs to determine effective material properties as a function of aggregate volume fraction, as was done in previous work (in 
2D), see Nilenius et al. [11]. The effect of ITZ on the numerical results will further be investigated so that the model can be calibrated with experimental data. Additionally, both Dirichlet and Neumann boundary conditions will be applied to obtain upper and lower bounds, respectively, on the effective material properties.

\section{References}

[1] Asahina, D., Bolander, J.E.: Voronoi-based discretizations for fracture analysis of particulate materials. Powder Technology 213(1-3), 92-99 (2011) ISSN 00325910 , http://linkinghub.elsevier.com/retrieve/pii/S0032591011003330, doi:10.1016/j.powtec.2011.07.010 252

[2] Bentz, D.P., Garboczi, E.J., Snyder, K.A.: National Institute of Standards, and Technology (U.S.). A hard core/soft shell microstructural model for studying percolation and transport in three-dimensional composite media. U.S. Dept. of Commerce, Technology Administration, National Institute of Standards and Technology (1999) 252

[3] de Berg, M., Cheong, O., van Kreveld, M., Overmars, M.: Computational Geometry: Algorithms and Applications, 3rd edn. Springer-Verlag TELOS, Santa Clara (2008) ISBN 3540779736, 9783540779735 253

[4] Caballero, A., Lopez, C., Carol, I.: 3D meso-structural analysis of concrete specimens under uniaxial tension. Comput. Methods Appl. Mech. Engrg. 195(52), 7182-7195 (2006) ISSN 00457825, doi:10.1016/j.cma.2005.05.052 252]

[5] Garboczi, E.: Multiscale Analytical/Numerical Theory of the Diffusivity of Concrete. Adv. Cem. Based Mater. 8(2), 77-88 (1998) ISSN 10657355, doi:10.1016/S1065-7355(98)00010-8 252

[6] Hain, M., Wriggers, P.: Numerical homogenization of hardened cement paste. Computational Mechanics 42(2), 178-7675 (2007) ISSN 0178-7675, doi:10.1007/s00466-007-0211-9 252

[7] Hain, M., Wriggers, P.: Computational homogenization of micro-structural damage due to frost in hardened cement paste. Finite. Elem. Anal. Des. 44(5), 233-244 (2008) ISSN 0168874X, doi:10.1016/j.finel.2007.11.020 252

[8] Hedenblad, G.: Moisture permeability of mature concrete, cement mortar and cement paste. PhD thesis, Div. of Building Materials LTH, Lund university (1993) 252

[9] Idiart, A.E., López, C.M., Carol, I.: Chemo-mechanical analysis of concrete cracking and degradation due to external sulfate attack: A meso-scale model. Cement and Concrete Composites 33(3), 411-423 (2011) ISSN 09589465, http://linkinghub.elsevier.com/retrieve/pii/S0958946510001939, doi:10.1016/j.cemconcomp.2010.12.001 249

[10] Kim, S., Al-rub, R.K.A.: Meso-scale computational modeling of the plasticdamage response of cementitious composites. Cement and Concrete Research 41(3), 8-8846 (2011) ISSN 0008-8846, doi:10.1016/j.cemconres.2010.12.002 249 
[11] Nilenius, F., Larsson, F., Lundgren, K., Runesson, K.: Macroscopic diffusivity in concrete determined by computational homogenization. Int. J. Numer. Anal. Meth. Geomech. (2012), doi:10.1002/nag.2097 258

[12] Ostoja-Starzewski, M.: Material spatial randomness: From statistical to representative volume element. Probabilistic Engineering Mechanics 21(2), 112-132 (2006) ISSN 02668920, http://linkinghub.elsevier.com/retrieve/pii/S0266892005000433 doi:10.1016/j.probengmech.2005.07.007 250

[13] Wang, L., Ueda, T.: Mesoscale modeling of water penetration into concrete by capillary absorption. Ocean Engineering 38(4), 519-528 (2011) ISSN 00298018, doi:10.1016/j.oceaneng.2010.12.019 249 